\title{
Dimostrazione d'un teorema sopra i massimi e minimi delle funzioni di più variabili in- dipendenti.
}

\author{
[Di Cosare Russyan, " Kharkoff (Russia).]
}

Nella presente Nota vien data una dimostrazione dol teorema seguente della teoria dei massimi e minimi telle funzioni di più variabili indipendenti: «Se per un sistema di valori $x=a, y=b, \ldots, z=c$ delle variabili indipen\& denti $x, y, \ldots, z$ si annullano tutte le derivate della funzione data $f(x, y, \ldots, z)$ «fino a quelle dell'ordine $2 n-1$ inclusive; se la forma

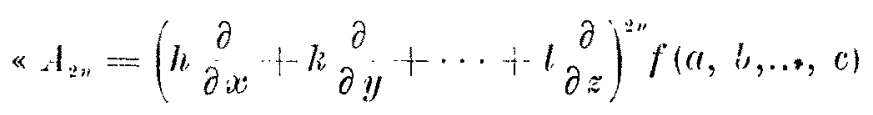

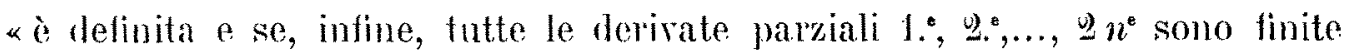
« e contimue in un intorno qualunque del punto considerato $x=a, y=b, \ldots$, $\ll z=c$, allora la funzione data $f(x, y, \ldots, z)$ ha nello stesso punto un mas«simo o un minimo. »

La dimostrazione di questo teorema, lata dal sig. U. JJNI $\left({ }^{*}\right)$, è basata. sulla proprieta del polinomio

$$
\left(\begin{array}{c}
\partial \\
\partial x
\end{array}+\frac{k}{h} \frac{\partial}{\partial y}+\cdots+\frac{l}{h} \frac{\partial}{\partial z}\right)^{2 \mu} f(\alpha, b, \ldots, c)
$$

di arere il limite inferiore del suo valore assoluto diverso da zero in tutto il campo infunito di valori delle variabili indipendenti $\frac{l}{h}, \ldots, \frac{l}{h}$, perciò è possibile di dimostrare l'esistenza dell'intorno del punto. $x=a, y=b, \ldots$,

(*) Lpsioni di cunclisi infinitesinnule antogralate a Pisa nel 1877 e pubblicate a Pisa nel 1907 . 
$z=c$ abbastanza piccolo, nei punti del quale il resto

$$
R_{2 n}=\frac{1}{2 n !}\left(h \frac{\partial}{\partial x}+k \frac{\partial}{\partial y}+\cdots+l \frac{\partial}{\partial z}\right)^{2 n} f(a+\theta h, b+\theta k, \ldots, c+\theta l)
$$

sia diverso da zero per tutti i sistemi di valori di $h, k, \ldots, l$, che non siano tutti nulli, ed abbia sempre il segno di $A_{2 n}$.

L'altra dimostrazione, data dal Grocciri, è basata sul teorema che il rapporto di due forme qualunque del medesimo grado pari, essendo il denominatore una forma definita, ha ambedue i limiti finiti, sotto la supposizione che le variabili delle forme non siano tutte nulle. Questo teorema è dimostrato per mezzo del cangiamento delle variabili indipendenti delle forme:

$$
x=x_{1} \overline{x^{2}+y^{2}+\cdots+z^{2}}, \ldots z=z_{1} \sqrt{x^{2}+y^{2}+\cdots+z^{2}} .
$$

Ma cionondimeno io credo sia utile di dare ancora una dimostrazione dello stesso teorema, perchè questa è, a mio credere, molto semplice.

Si ha così a dimostrare, che se tutte le condizioni del teorema suindicato sono soddisfatte, esiste sempre un numero positivo $\delta$, sufficientemente piccolo, tale che per

$$
x-a,<\delta, \ldots, z-\cdots c \delta
$$

la differenza

$$
f(x, y, \ldots, z)-f(n, b, \ldots, c)
$$

non muta mai il segno.

A causa delle ipotesi fatte sulle derivate $1 . ;, 2 . ; 3,{ }^{\circ}, \ldots,(2 n)^{\bullet}$ della funzione data nell'intorno dato del punto $x=a, y=b, \ldots, z=c$ esiste per gli stessi valori di variabili $x, y, \ldots, z$ la formola di TAYLoR:

$$
\begin{gathered}
f(x, y, \ldots, z)-f(a, b, \ldots, c)= \\
=\frac{1}{2 n !}\left[(x-a) \frac{\partial}{\partial x}+\cdots+(z-c) \frac{\partial}{\partial z}\right]^{2 n} f[a+\theta(x-a), \ldots, c+\theta(z-c) \mid,
\end{gathered}
$$

$o$, indicando per brevità le differenze $x-a, \ldots, \approx-c$ con $h, \ldots, l$, la formola

$$
\begin{gathered}
f(a+-h, b+k, \ldots, c+l)-f(a, b, \ldots, c)= \\
=\frac{1}{2 n !}\left[h \frac{\dot{\partial}}{\partial x}+k \frac{\partial}{\partial y}+\cdots+l \frac{\partial}{\partial z}\right]^{2 \prime \prime} f(a+\theta h, b+\theta k, \ldots, c+\theta l) .
\end{gathered}
$$


Esaminiamo il segno del resto

$$
R_{z n}=\frac{1}{2 n !}\left(h \frac{\partial}{\partial x}+k \frac{\partial}{\partial y}+\cdots+l \frac{\partial}{\partial z}\right)^{2 n} f(a+\theta h, b+\theta k, \ldots, c+\theta l)
$$

per i valori considerati di $h, k, \ldots, l$, che non sono tutti nulli.

Noi abbiamo evidentemente

$$
R_{3 n}=\frac{1}{2 n !} A_{2 n}\left(1+\frac{2 n ! R_{2 n}-A_{2 n}}{A_{2 n}}\right) .
$$

Consideriamo il rapporto

che è uguale a

$$
\frac{2 n ! R_{2 n}-A_{2 n}}{A_{2 n}}
$$

$$
\frac{\left(h \frac{\partial}{\partial x}+k \frac{\partial}{\partial y}+\cdots+l \frac{\partial}{\partial z}\right)^{2 n}[f(a+\theta l, b+\theta k, \ldots, c+\theta l)-f(a, b, \ldots, c)]}{\left(h \frac{\partial}{\partial x}+k \frac{\partial}{\partial y}+\cdots+l \frac{\partial}{\partial z}\right)^{2, \prime} f(a, b, \ldots, c)},
$$

e cerchiamo il suo ralore assoluto.

Cangiamo perciò le rariabili $h, k, \ldots, l$ nel molo seguente. Sia $p$ un numero positivo qualunque, dato a piacere; sia poi $I$ il più grande fra i valori assoluti delle rariabili $h, k, \ldots, l$, e sia, p. es., $L=\vdots l$. Poniamo

Ne risulta, che

$$
h={ }_{p}^{L} h_{1}, \quad k={ }_{p}^{T} k_{1}, \ldots, \quad l={ }_{p}^{T} l_{1} .
$$

$$
h_{1}|\leq p, \quad| k_{1} \mid \leq p, \ldots, \quad \text { con } \mid l_{1} !=p
$$

e così uno almeno fra $\mathrm{i}$ valori assoluti delle nuove variabili è uguale a $p$; gli altri non superano $p$. Perciò tutto il campo dei valori delle variabili $h_{1}$, $k_{1}, \ldots, l_{1}$ può ottenersi nel modo seguente: ogni variabile $h_{1}, k_{1}, \ldots, l_{1}$ considerata separatamente e successivamente riceve $\mathrm{i}$ valori $\pm p$, mentre le altre ricevono allora ciascuna un continuo di valori nell'intervallo $(-p, p)$.

Dopo questo cangiamento si avrà :

$$
\begin{aligned}
& \frac{2 n ! R_{3_{n}}-A_{2 n}}{J_{2 n}}=
\end{aligned}
$$

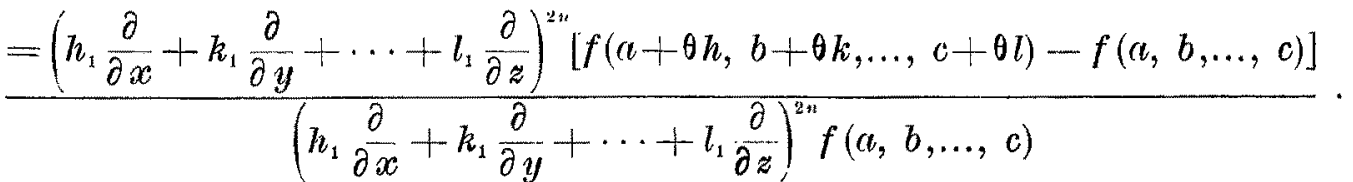




\section{Russyan: Dimostrazione d'un teorema sopra $i$ massimi e minimi}

Indichiamo la forma definita nel denominatore con $A_{2 n}^{\prime}$. Si può dimostrare che esiste un numero positivo $P_{2 n}$ tale, che

$$
\left|A_{2 n}^{\prime}\right|>P_{y_{n}}
$$

per tutti i valori considerati delle variabili $h_{1}, k_{1}, \ldots, l_{1}$.

Infatti, se la forma $d_{2 n}^{\prime}$ potesse prendere nel campo considerato valori numericamente piccoli a piacere, in virtù della sua continuità essa dovrebbe annullarsi, il che è impossibile, poichè la forma $A_{2 n}^{\prime}$ è definita ed una almeno delle variabili $h_{1}, k_{1}, \ldots, l_{1}$ ha il valore $\pm p$ diverso da zero.

Sia allora $n$ il più grande dei valori assoluti dei moltiplicatori dei coefficienti di $h_{1}, k_{1}, \ldots, l_{1}$ nel numeratore, che hanno tutti la forma

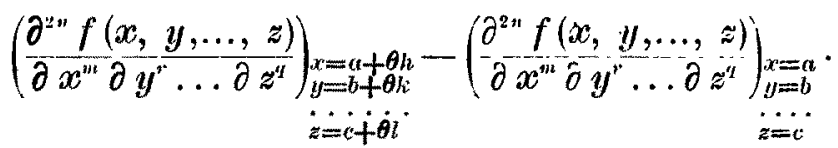

Allora si avrà evidentemente

$$
\left.\mid \frac{2 n ! R_{2 n}-A_{2 n}}{A_{2 n}}<\frac{\rho^{2 n} p^{2 n}}{P_{2 n}} n_{i}^{2}{ }^{*}{ }^{*}\right)
$$

dove $p$ è il numero delle variabili $x, y, \ldots, z ; p, P_{2 *}$ sono costanti, ma $h$ è variabile con $h, k, \ldots, l$. Ora le derivate dell'ordine $2 n$ sono continue e finite nel punto $x=a, y=b, \ldots, z=c$; esiste perciò un numero positivo $\delta$, abbastanza piccolo, tale che per

si hat

$$
h<\delta, \quad ; k<\delta, \ldots, \quad: l<\delta
$$

$$
n<\frac{P_{2,1}}{\rho^{2 n} p^{2,11}} .
$$

Allora per gli stessi valori di $h, k, \ldots, l$, ma che non siano tutti nulli,

(*) Si potrebbe dimostrare in modo simile che nel caso di due forme qualunque $A_{2 n}, b_{2 n}$ del medesimo grado pari, dove $A_{2 n}$ è la forma definita, si ha sempre

$$
\left|\begin{array}{c}
B_{2^{n}} \\
\bar{A}_{2^{n}}
\end{array}\right|<\left(\begin{array}{c}
2 n+\rho-1 \\
\rho-1
\end{array}\right) \frac{p^{2 n}}{P_{2^{n}}^{n}} n
$$

dove $n$ ¿̀ il più grande dei valori assoluti dei coefficienti costanti della forma $B_{2^{n}}$. 
avremo che

ed il resto

$$
\frac{2 n ! R_{n n}-A_{2 n}}{A_{2 n}} \mid<1
$$

$$
R_{2 n}=\frac{1}{2 n !} A_{2 n}\left(1+\frac{2 n ! K_{2 n}-A_{2 n}}{A_{2 n}}\right)
$$

sarà diverso da zero ed arrà il segno costaute, identico con quello della forma detinita $A_{2 n}$.

Noi abbiamo dunque dimostrato che esiste un numero positivo $\delta$ lale, che per tutti i valori di $x, y, \ldots, z$ nell'intorno dato, pei quali

la differenza

$$
|x-a|<\delta, \quad \mid y-b<\delta, \ldots, \quad z-c:<\delta
$$

$$
f(x, y, \ldots, z)-f(a, b, \ldots, c)
$$

sarà diversa da zero quando una almeno della differenza $x-a, y-b, \ldots$, $z--c$ non è zero, ed avrà il segno costante, identico con (juello della forma definita $A_{2,}$, cioè che la funzione data $f(x, y, \ldots, z)$ ha per $x=a, y=b, \ldots$, $z=c$ un massimo o un minimo. 